\title{
Commuting operations on signal-flow graphs for visualization of interconnected systems
}

\author{
Andreas Johansson* \\ * Control Engineering Group \\ Luleå University of Technology \\ SE-971 87 Luleå, Sweden \\ Email: andreas.johansson@ltu.se \\ Phone +46920492334
}

\begin{abstract}
We explore the signal flow graph as a setup for visualizing and analyzing interconnected systems. Two operations for facilitating interpretation of signal flow graphs are defined. These are hiding of unimportant nodes and elimination of self-references and it is shown that the operations commute under some conditions and that the hiding operation preserves the physical structure of the system in a certain sense. Also, the Loop Gain Index (LGI) is introduced as a measure of the relative importance of the edges in terms of dynamic behavior of the interconnected system. The LGI is illustrated with a numerical example.
\end{abstract}

\section{INTRODUCTION}

A modern process industry is a very complex system where hundreds or even thousands of variables are connected through dynamic systems. Examples of such interconnections are material flows and reflows. Other examples are connections through supply grids for e.g. pressurized air. Adding to this control loops on both low and high level may give a system with unintelligible causality and unpredictable dynamics.

For the control engineer, these very complex interconnected systems are a challenge. The question is how to represent and visualize the complexity in a comprehensible way and how to analyze it regarding e.g. control structure selection and dynamic behavior. Computerized tools for visualization of complex system are e.g. Matlab Simulink and Dymola, the latter based on the generic modelling language Modelica. However, these are mainly intended for simulation and do not support the desired analysis tools.

A directed graph is a highly abstract way of visualizing complexity in various applications. By letting the nodes represent signals and the edges linear dynamic systems, one obtains the signal flow graph (Mason (1953)), which is a very general and abstract representation for interconnected, dynamic, linear systems. Compared to a block diagram, the signal flow graph has the advantage of being closely related to an algebraic representation, in terms of matrices consisting of the edges in the graph. Another advantage with the signal flow graph is that it allows choosing the level of detail in the representation, thus encompassing both the input/output form as well as the state-space form as special cases. The former is obtained by hiding every variable that is not an input or output and the latter by having a node for each state variable with integrators as edges. Tools for decomposing interconnected systems in state-space form are given in e.g. Sezer and Šiljak (1986); Zečević and Šiljak (2005). Another special case is considered in Castaño and Birk (2009) where an autoregressive (AR) structure of the edges is assumed.

Here, we shall assume the edges to be general, linear, dynamic systems. The advantage with this is that we may consider also infinite-dimensional systems as edges, such as time-delays and other distributed-parameter processes. These are very common in the process industry in the form of conveyor belts and tubular reactors, such as blast furnaces and paper pulp digesters (Ding et al. (2007)). Application to time-varying and nonlinear systems is conceivable but then the results will be valid for a specific point in time or a specific working point.

What we seek to develop here are operations and analysis tools on signal flow graphs. The main operation will be hiding of nodes that are not presently relevant, without changing the dynamics. This will enable us to choose the abstraction level of the visualization of a plant, on a range from the detailed state-space representation to the abstract input/output form.

The analysis tools will be aimed at measuring the strength of each edge, in different regards. A basic measure is an operator norm taken on each edge but, as we shall see in an example, this quantity does not give deep insight into the properties of the system. An improvement is to weigh this quantity relative to the sum of the norms of all the edges entering or leaving a node, (Castaño and Birk (2009)). Here, we propose a measure, termed Loop Gain Index (LGI), targeting the importance of each edge in terms of the input/output behavior for simulation purposes. The interconnected system considered can then be taken to be the whole closed loop plant, including controllers and the input signals are then external signals, such as ambient variables, raw material qualities and setpoints provided by operators. 
An important application of such measures are for clustering of the interconnected system, i.e. grouping nodes that are strongly connected. Such a grouping can facilitate interpretation the interconnected system as well as the application of numerical algorithms such as simulation or controller design. Clustering algorithms found in Sezer and Šiljak (1986); Zečević and Siljak (2005) are formulated for state-space systems but may also be applied to general, weighted graphs.

Another application of the measures is for system simplification, i.e. to support decisions on which edges may be neglected or replaced by an approximation. Note the significant difference from the concept of model reduction, which has the sole purpose of reducing the model order. Neglecting an edge does not necessarily decrease the order of the underlying state-space representation. Simple examples show that it may even increase. However, neglecting an edge may considerably simplify various practical aspects of the control design. Unless fault tolerance is addressed specifically, then limiting, if possible, the number of signals that the controller depends on may be advantageous for making the controller tolerant to sensor faults and shutdown of subprocesses, etc.

\subsection{Notation and preliminaries}

The identity matrix is denoted $I$. A vector or matrix where each element is equal to one or zero is denoted 1 or 0 , respectively. For clarity, the dimension of $I, 1$, and 0 is sometimes signified with an index, e.g. $I_{n}$ is the $n \times n$ identity matrix and $1_{n}$ is a vector of $n$ elements, each equal to 1 . The notation $\circ$ signifies the element-wise product (known as Hadamard or Schur product). A permutation matrix $P$ is a square matrix with exactly one element equal to 1 in each row and column and all other elements equal to 0 . Permutation matrices are orthogonal which means that $P^{-1}=P^{T}$.

Linear time-invariant systems defined by convolution by an impulse response are written in bold-face font, i.e. $y=\mathbf{G} u$ means that $y(t)=\int_{0^{-}}^{\infty} G(t-\tau) u(\tau) \mathrm{d} \tau$ where $G$ is the impulse response of $\mathbf{G}$. In the sequel, every system is assumed to belong to the space $\mathrm{A}_{e}$ which means that its impulse response can be written as

$$
G(t)=G_{a}(t)+G_{0} \delta(t)+\sum_{i=1}^{\infty} G_{i} \delta\left(t-t_{i}\right), t \geq 0
$$

where $\delta(t)$ is the Dirac delta distribution, $0<t_{1}<$ $t_{2}<\ldots$ and $G_{a} \in \mathrm{L}_{1 e}$, i.e. $\int_{0^{-}}^{T}\left|G_{a}(t)\right| \mathrm{d} t<\infty$ for any finite $T>0$. A non-dynamic linear systems (i.e. matrix multiplication), corresponding to the impulse response $A \delta$ is associated with an operator denoted $A$. In this manner, the unit operator is denoted $I$ (or 1 for scalarvalued signals). Inverse operators are denoted with ${ }^{-1}$, i.e. $\mathbf{G G}^{-1}=\mathbf{G}^{-1} \mathbf{G}=I$ and for scalar operators, the notation $\mathbf{G}^{-1}=1 / \mathbf{G}$ is sometimes used. Laplace transforms of signals are denoted with the same symbol as the signal itself. When necessary, the argument $(s)$ is used to avoid confusion.

The lower LFT (Linear Fractional Transformation) is defined as usual as (Skogestad and Postlethwaite (2005))

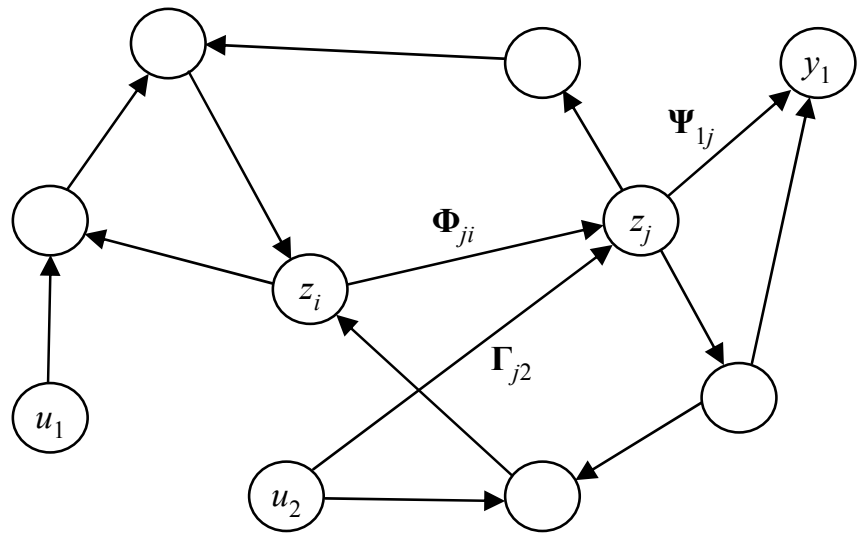

Fig. 1. A graph for representing an interconnected system. The nodes are signals and the edges are linear, dynamic systems

$$
\mathcal{F}\left(\left[\begin{array}{ll}
\mathbf{P}_{11} & \mathbf{P}_{12} \\
\mathbf{P}_{21} & \mathbf{P}_{22}
\end{array}\right], \mathbf{K}\right)=\mathbf{P}_{11}+\mathbf{P}_{12} \mathbf{K}\left(I-\mathbf{P}_{22} \mathbf{K}\right)^{-1} \mathbf{P}_{21}
$$

assuming that $\mathbf{P}_{22}$ and $\mathbf{K}^{T}$ have the same dimension.

Lemma 1.

$$
\begin{aligned}
& \text { (i) } \mathcal{F}\left(\left[\begin{array}{cc}
\mathbf{A} & 0 \\
0 & \mathbf{B}
\end{array}\right] \mathbf{P}\left[\begin{array}{cc}
\mathbf{C} & 0 \\
0 & \mathbf{D}
\end{array}\right], \mathbf{K}\right)=\mathbf{A} \mathcal{F}(\mathbf{P}, \mathbf{D K B}) \mathbf{C} \\
& \text { (ii) } \mathcal{F}\left(\mathcal{F}\left(\mathbf{P}, \mathbf{K}_{1}\right), \mathbf{K}_{2}\right)=\mathcal{F}\left(\mathbf{P},\left[\begin{array}{cc}
\mathbf{K}_{2} & 0 \\
0 & \mathbf{K}_{1}
\end{array}\right]\right)
\end{aligned}
$$

Proof. The lemmas are straightforward to verify using a representation of the LFT as the input/output operator from $w$ to $z$ in

$$
\begin{aligned}
z & =\mathbf{P}_{11} w+\mathbf{P}_{12} u \\
v & =\mathbf{P}_{21} w+\mathbf{P}_{22} u \\
u & =\mathbf{K} v
\end{aligned}
$$

but the details are omitted for sake of brevity.

\section{SIGNAL FLOW GRAPHS}

To represent interconnected systems, we will use the signal-flow graph which consists of nodes that represent the signals and edges that describe how the signals affect each other, see Fig. 1. Three categories of nodes are considered, input signals $u_{i}, i=1 . . p$, output signals $y_{i}, i=1 . . q$, and internal signals $z_{i}, i=1 . . n$. The interpretation of these categories may vary depending on the problem at hand. The outputs may be a subset of the internal variables, e.g. the measurement signals or the controlled variables but it presents no added difficulty to allow general dynamical relations between the internal variables and the outputs.

The internal signals are assumed to be affected linearly by the input signals and the other internal signals, i.e.

$$
z_{i}=\boldsymbol{\Phi}_{i 1} z_{1}+\ldots+\boldsymbol{\Phi}_{i n} z_{n}+\boldsymbol{\Gamma}_{i 1} u_{1}+\ldots+\boldsymbol{\Gamma}_{i p} u_{p}
$$

for $i=1 . . n$ where $\boldsymbol{\Phi}_{i j}$ and $\boldsymbol{\Gamma}_{i j}$ are linear dynamic systems. Similarly, the output is assumed to be affected linearly by the internal signals and the inputs as

$$
y_{i}=\boldsymbol{\Psi}_{i 1} z_{1}+\ldots+\boldsymbol{\Psi}_{i n} z_{n}+\boldsymbol{\Omega}_{i 1} u_{1}+\ldots+\boldsymbol{\Omega}_{i p} u_{p}
$$

for $i=1 . . q$. By collecting the signals $u_{i}, z_{i}$, and $y_{i}$ into vectors $u, z$, and $y$ and defining the multivariable, dynamic systems $\boldsymbol{\Phi}, \boldsymbol{\Gamma}, \boldsymbol{\Psi}$, and $\boldsymbol{\Omega}$ whose $i, j$ th element are 
$\boldsymbol{\Phi}_{i j}, \boldsymbol{\Gamma}_{i j}, \boldsymbol{\Psi}_{i j}$, and $\boldsymbol{\Omega}_{i j}$, respectively, the signal flow graph representation may now be formulated as

$$
\begin{aligned}
& z=\boldsymbol{\Phi} z+\boldsymbol{\Gamma} u \\
& y=\boldsymbol{\Psi} z+\boldsymbol{\Omega} u
\end{aligned}
$$

It is straightforward to see that the internal variables are related to the inputs as $z=(I-\mathbf{\Phi})^{-1} \boldsymbol{\Gamma} u$ and that the input/output relation is

$$
y=\left(\boldsymbol{\Psi}(I-\boldsymbol{\Phi})^{-1} \boldsymbol{\Gamma}+\boldsymbol{\Omega}\right) u
$$

A more usual way of representing interconnected systems is Vidyasagar (1980)

$$
\begin{aligned}
e & =u-\mathbf{H} y \\
y & =\mathbf{G} e
\end{aligned}
$$

but we will use the form (4) instead because of its more direct relation to the graph representation.

Clearly, questions of existence and uniqueness of a solution to (4) only concerns the operator $\boldsymbol{\Phi}$. Using the definition from Vidyasagar (1980), we will say that the system $z^{\prime}=$ $\boldsymbol{\Phi} z^{\prime}+u^{\prime}$ is well-posed if (i) there is a unique solution $z^{\prime}$, given an input $u^{\prime}$, (ii) the solution $z^{\prime}$ depends causally on $u^{\prime}$, and (iii) the dependence of $z^{\prime}$ on $u^{\prime}$ is continuous. For details of the definition, see Vidyasagar (1980), where a necessary and sufficient condition for well-posedness is given. Assuming that the impulse response of the elements of $\boldsymbol{\Phi}$ is of the form (1), i.e.

$$
\Phi(t)=\Phi_{a}(t)+\Phi_{0} \delta(t)+\sum_{i=1}^{\infty} \Phi_{i} \delta\left(t-t_{i}\right), t \geq 0
$$

then $z^{\prime}=\boldsymbol{\Phi} z^{\prime}+u^{\prime}$ is well-posed if and only if $\operatorname{det}(I+$ $\left.\Phi_{0}\right) \neq 0$. For our purposes, it is sufficient to note that well-posedness of $z^{\prime}=\mathbf{\Phi} z^{\prime}+u^{\prime}$ implies existence of a unique operator $(I-\boldsymbol{\Phi})^{-1}$. Here, we will also introduce the concept of well-posedness of a subsystem.

Definition 1. Consider an interconnected system (4) and a subset $\mathrm{N}$ of its nodes. Let $E$ be a matrix that picks out the internal variables $z_{\mathrm{N}}$ corresponding to $\mathrm{N}$ from the vector $z$, i.e. $z_{\mathrm{N}}=E z$. Then, the subsystem associated with the nodes $\mathrm{N}$ is said to be well-posed if the system $z^{\prime}=E \boldsymbol{\Phi} E^{T} z^{\prime}+u^{\prime}$ is well-posed.

\section{VISUALIZATION}

Here we introduce the concept of visualization, by which we mean the representation of a linear system to show "internal wirings" in the form of a signal-flow graph.

Definition 2. Given a linear, multivariable system $\mathbf{H}$, the quadruple $(\boldsymbol{\Phi}, \boldsymbol{\Gamma}, \boldsymbol{\Psi}, \boldsymbol{\Omega})$ is called a visualization of $\mathbf{H}$ if $\boldsymbol{\Psi}(I-\boldsymbol{\Phi})^{-1} \boldsymbol{\Gamma}+\boldsymbol{\Omega}=\mathbf{H}$.

In the sequel, the equivalent representation

$$
\mathbf{Q}=\left[\begin{array}{ll}
\boldsymbol{\Omega} & \boldsymbol{\Psi} \\
\Gamma & \boldsymbol{\Phi}
\end{array}\right]
$$

will also be used. The kinship to the concept of realization should be clear. Indeed, given a realization of the system $\mathbf{G}$ as $G(s)=C(s I-A)^{-1} B+D$, a visualization with the internal variables $z$ chosen as the state variables of the realization is given by $\Phi(s)=A / s, \Gamma(s)=B / s, \Psi(s)=C$, and $\Omega(s)=D$. A realization can thus be considered as an extreme case of a visualization, where all the state variables are visible. On the other extreme, we have the (a) (b)
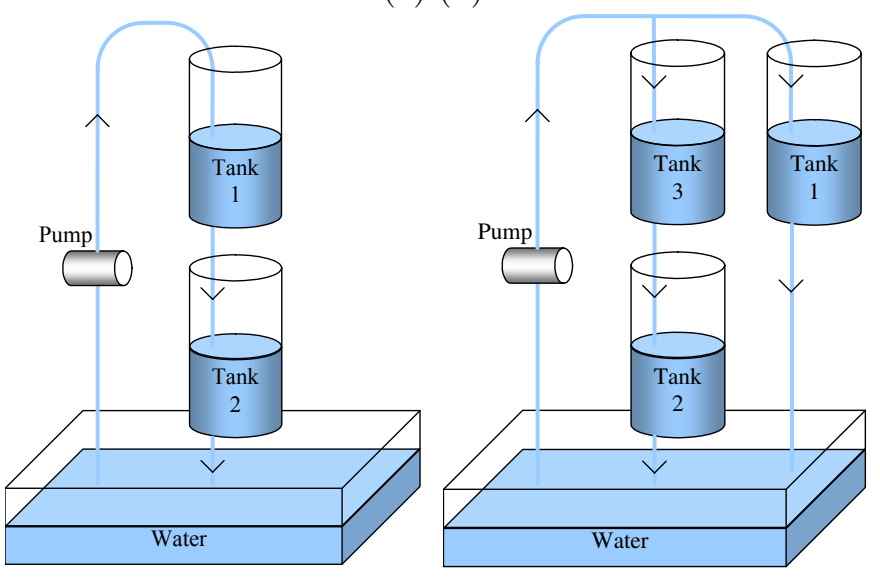

Fig. 2. (a) The water tank process considered in Example 1. (b) another physical system with the same values of the internal variables

input/output visualization obtained by the trivial choice $\Omega(s)=G(s)$ and $\Gamma(s), \Phi(s), \Psi(s)$ equal to zero.

Obviously, a visualization (like a realization) is not unique. However, unlike a realization, which is unique given a minimal set of state variables, a visualization is not unique even if the internal variables are chosen, as the following example shows.

Example 1. Consider the simple example of a double water tank process as illustrated in Fig. 2(a) where the control signal to the pump is the input $u$ while the water levels in the identical Tank 1 and Tank 2 are the internal variables $z_{1}$ and $z_{2}$, respectively. The inflow to Tank 1 is assumed to be proportional to $u$ and the outflow from both tanks are assumed proportional to the water levels. This results in the process model

$$
\begin{aligned}
& z_{1}(s)=\frac{k}{s T+1} u(s) \\
& z_{2}(s)=\frac{1}{s T+1} z_{1}(s)
\end{aligned}
$$

for some constants $k$ and $T$. The process model above corresponds to the visualization $(\boldsymbol{\Phi}, \boldsymbol{\Gamma}, \boldsymbol{\Psi}, \boldsymbol{\Omega})$ with

$$
\Phi(s)=\left[\begin{array}{cc}
0 & 0 \\
\frac{1}{s T+1} & 0
\end{array}\right], \quad \Gamma(s)=\left[\begin{array}{c}
\frac{k}{s T+1} \\
0
\end{array}\right]
$$

and $\Psi(s), \Omega(s)$ arbitrary since we are not concerned with the outputs in this example. Equivalently, we may substitute (5) into (6) and obtain

$$
\begin{aligned}
& z_{1}(s)=\frac{k}{s T+1} u(s) \\
& z_{2}(s)=\frac{k}{(s T+1)^{2}} u(s)
\end{aligned}
$$

i.e. the same response of the internal variables to the input but with the visualization $\left(\boldsymbol{\Phi}^{\prime}, \boldsymbol{\Gamma}^{\prime}, \boldsymbol{\Psi}, \boldsymbol{\Omega}\right)$ with

$$
\Phi^{\prime}(s)=\left[\begin{array}{ll}
0 & 0 \\
0 & 0
\end{array}\right], \quad \Gamma^{\prime}(s)=\left[\begin{array}{c}
\frac{k}{s T+1} \\
\frac{k}{(s T+1)^{2}}
\end{array}\right]
$$

which rather corresponds to the tank setup in Fig. 2(b). The pump is here assumed to have double capacity and distribute the water equally to the identical Tank 1 and 
Tank 3. Thus, the water levels in Tank 1 and Tank 2 will be the same as in Fig. 2(a), but there is no longer any physical influence on Tank 2 from Tank 1 . The important point that is stressed with this example is that although visualizations are nonunique, some visualization may be consistent with the process physics while others are not.

\section{OPERATIONS ON SIGNAL FLOW GRAPHS}

When visualizing an interconnected system, the critical issue is abstraction, i.e. the ability to disregard unnecessary details. We envision a computerized tool, with a graphical interface showing a process industry facility in the form of a signal flow graph. In this tool, we can choose the level of abstraction so that when dealing with supervisory control loops, internal variables of low level loops can be hidden. Also, we may e.g. want to hide state-variables of high-order black-box models. Thus, the central operation that will be defined is the hiding of a set $\mathrm{N}$ of nodes that are not presently important, without changing the relation between the inputs and the remaining internal variables. Let $\mathrm{N}^{C}$ denote the remaining nodes. We may now state

Definition 3. Consider a visualization $(\boldsymbol{\Phi}, \boldsymbol{\Gamma}, \boldsymbol{\Psi}, \boldsymbol{\Omega})$ of $\mathbf{G}$ with internal variables $z$ and let $\mathrm{N}$ be a subset of the nodes and $\mathrm{N}^{C}$ the remaining nodes corresponding to the internal variables $z^{\prime}=E z$. Then the operation $\mathcal{H}_{\mathrm{N}}$ is a hiding operation for $\mathrm{N}$ if $(\hat{\boldsymbol{\Phi}}, \hat{\boldsymbol{\Gamma}}, \hat{\boldsymbol{\Psi}}, \hat{\boldsymbol{\Omega}})=\mathcal{H}_{\mathrm{N}}(\boldsymbol{\Phi}, \boldsymbol{\Gamma}, \boldsymbol{\Psi}, \boldsymbol{\Omega})$ is a visualization of $\mathbf{G}$ and $E(I-\boldsymbol{\Phi})^{-1} \boldsymbol{\Gamma}=(I-\hat{\mathbf{\Phi}})^{-1} \hat{\boldsymbol{\Gamma}}$.

As shown in Example 1, this requirement is not sufficient for the reduced system (i.e. with the nodes $\mathrm{N}$ hidden) to be unique. Therefore, we will also require the hiding operation to preserve the physical structure of the system. By this we shall mean that a perturbation introduced in one of the nodes of $\mathrm{N}^{C}$ will give the same response in the nodes of the reduced system as in the nodes $\mathrm{N}^{C}$ of the original system. Introducing a perturbation in one of the nodes of $\mathrm{N}^{C}$, say $z_{i}$, can be accomplished by introducing an auxiliary input $u_{i}^{\prime}$ and attaching it to $z_{i}$ using an edge with a unit operator. The response to this input in the nodes in $\mathrm{N}^{C}$ of the original system is $E(I-\boldsymbol{\Phi})^{-1} e_{i} u_{i}^{\prime}$. Thus, we may give the following definition.

Definition 4. Consider a visualization $(\boldsymbol{\Phi}, \boldsymbol{\Gamma}, \boldsymbol{\Psi}, \boldsymbol{\Omega})$ with internal variables $z$ and let $\mathrm{N}$ be a subset of the nodes and $\mathrm{N}^{C}$ the remaining nodes corresponding to the internal variables $z^{\prime}=E z$. Then the hiding operation $\mathcal{H}_{\mathrm{N}}$ is said to preserve the physical structure if $E(I-\mathbf{\Phi})^{-1} E^{T}=(I-$ $\hat{\mathbf{\Phi}})^{-1}$.

Note that the two visualizations in Example 1 have different physical structure in this sense, since a perturbation introduced in $z_{1}$ affects the variable $z_{2}$ in the visualization (7) but not in (8).

Finally, we want the hiding operation to be commutative, i.e. independent of order. To be specific, we mean that hiding a subset $\mathrm{N}$ of the nodes and then hiding another subset $\mathrm{M}$ should give the same visualization as first hiding the subset $\mathrm{M}$ and then the subset $\mathrm{N}$.

Definition 5. The hiding operation $\mathcal{H}$ is said to be commutative if $\mathcal{H}_{\mathrm{M}} \mathcal{H}_{\mathrm{N}}=\mathcal{H}_{\mathrm{N}} \mathcal{H}_{\mathrm{M}}$

In this section we will define a hiding operation that satisfies the three definitions above. Also, we will define the operation of eliminating edges from a node to itself, and we will state that this operation commutes with the hiding operation in a certain sense.

\subsection{Linear transformation of internal variables}

For an invertible matrix $T$, a new set of internal variables may be defined as $\hat{z}=T z$ so that the new visualization $\hat{z}=T \boldsymbol{\Phi} z+T \boldsymbol{\Gamma} u=T \boldsymbol{\Phi} T^{-1} z+T \boldsymbol{\Gamma} u$ is obtained, i.e.

$$
\begin{aligned}
& \hat{z}=\hat{\boldsymbol{\Phi}} \hat{z}+\hat{\boldsymbol{\Gamma}} u \\
& y=\hat{\mathbf{\Psi}} \hat{z}+\boldsymbol{\Omega} u
\end{aligned}
$$

where

$$
\left[\begin{array}{cc}
\hat{\boldsymbol{\Omega}} & \hat{\mathbf{\Psi}} \\
\hat{\boldsymbol{\Gamma}} & \boldsymbol{\Phi}
\end{array}\right]=\left[\begin{array}{cc}
I_{q} & 0 \\
0 & T
\end{array}\right]\left[\begin{array}{cc}
\boldsymbol{\Omega} & \boldsymbol{\Psi} \\
\boldsymbol{\Gamma} & \boldsymbol{\Phi}
\end{array}\right]\left[\begin{array}{cc}
I_{p} & 0 \\
0 & T^{-1}
\end{array}\right]
$$

In particular, we will consider the case when $T$ is a permutation matrix, in which case $T^{-1}=T^{T}$ and the linear transformation only reorders the nodes thus preserving their physical interpretation.

\subsection{Hiding the last node}

We will first consider hiding the last node, i.e. $z_{n}$. Let us partition the interconnected system as

$$
\begin{aligned}
{\left[\begin{array}{l}
z^{1} \\
z_{n}
\end{array}\right] } & =\left[\begin{array}{ll}
\boldsymbol{\Phi}^{11} & \boldsymbol{\Phi}^{1 n} \\
\boldsymbol{\Phi}^{n 1} & \boldsymbol{\Phi}_{n n}
\end{array}\right]\left[\begin{array}{l}
z^{1} \\
z_{n}
\end{array}\right]+\left[\begin{array}{l}
\boldsymbol{\Gamma}^{1} \\
\boldsymbol{\Gamma}_{n}
\end{array}\right] u \\
y & =\left[\begin{array}{ll}
\boldsymbol{\Psi}^{1} & \boldsymbol{\Psi}_{n}
\end{array}\right]\left[\begin{array}{l}
z^{1} \\
z_{n}
\end{array}\right]+\boldsymbol{\Omega} u
\end{aligned}
$$

where $z^{1}=\left[\begin{array}{ll}I_{n-1} & 0\end{array}\right] z$ are all internal variables except $z_{n}$ and assume that the system $z^{\prime}=\boldsymbol{\Phi}_{n n} z^{\prime}+u^{\prime}$ is well-posed. Then we can express $z_{n}=\frac{1}{1-\boldsymbol{\Phi}_{n n}}\left(\boldsymbol{\Phi}^{n 1} z^{1}+\boldsymbol{\Gamma}_{n} u\right)$ which, substituted into the expression for $z^{1}$ gives

$$
\begin{aligned}
z^{1} & =\left(\boldsymbol{\Phi}^{11}+\frac{\boldsymbol{\Phi}^{1 n} \boldsymbol{\Phi}^{n 1}}{1-\boldsymbol{\Phi}_{n n}}\right) z^{1}+\left(\boldsymbol{\Gamma}^{1}+\frac{\boldsymbol{\Phi}^{1 n} \boldsymbol{\Gamma}_{n}}{1-\boldsymbol{\Phi}_{n n}}\right) u \\
y & =\left(\boldsymbol{\Psi}^{1}+\frac{\boldsymbol{\Psi}_{n} \boldsymbol{\Phi}^{n 1}}{1-\boldsymbol{\Phi}_{n n}}\right) z^{1}+\left(\boldsymbol{\Omega}+\frac{\boldsymbol{\Psi}_{n} \boldsymbol{\Gamma}_{n}}{1-\boldsymbol{\Phi}_{n n}}\right) u
\end{aligned}
$$

The above is a visualization of the reduced system, and we argue that this visualization is quite intuitively defined. Consider the case when $\boldsymbol{\Phi}_{n n}=0$, i.e. $z_{n}$ does not reference itself directly. Then this visualization is obtained by replacing the effect of node $i$ on node $j$ via node $n$ through edges $\boldsymbol{\Phi}_{n i}$ and $\boldsymbol{\Phi}_{j n}$ by the direct link $\boldsymbol{\Phi}_{j i}=$ $\boldsymbol{\Phi}_{j n} \boldsymbol{\Phi}_{n i}$. If $\boldsymbol{\Phi}_{n n} \neq 0$ then the only difference is that each of these new, direct links will obtain the factor $\frac{1}{1-\boldsymbol{\Phi}_{n n}}$. And if there already was a direct link from $i$ to $j$, then the new link is added to it.

Example 2. Consider (4) with the structure

$$
\boldsymbol{\Phi}=\left[\begin{array}{cccc}
0 & \boldsymbol{\Phi}_{12} & 0 & \boldsymbol{\Phi}_{14} \\
0 & 0 & 0 & 0 \\
0 & 0 & 0 & \boldsymbol{\Phi}_{34} \\
0 & \boldsymbol{\Phi}_{42} & \boldsymbol{\Phi}_{43} & 0
\end{array}\right], \quad \boldsymbol{\Gamma}=\left[\begin{array}{cc}
0 & 0 \\
0 & \boldsymbol{\Gamma}_{22} \\
0 & 0 \\
\boldsymbol{\Gamma}_{41} & 0
\end{array}\right]
$$

as illustrated in Fig. 3(a). After hiding the last node the visualization

$$
\boldsymbol{\Phi}^{\prime}=\left[\begin{array}{ccc}
0 & \boldsymbol{\Phi}_{12}+\boldsymbol{\Phi}_{14} \boldsymbol{\Phi}_{42} & \boldsymbol{\Phi}_{14} \boldsymbol{\Phi}_{43} \\
0 & 0 & 0 \\
0 & \boldsymbol{\Phi}_{34} \boldsymbol{\Phi}_{42} & \boldsymbol{\Phi}_{43} \boldsymbol{\Phi}_{34}
\end{array}\right], \boldsymbol{\Gamma}^{\prime}=\left[\begin{array}{cc}
\boldsymbol{\Phi}_{14} \boldsymbol{\Gamma}_{41} & 0 \\
0 & \boldsymbol{\Gamma}_{22} \\
\boldsymbol{\Phi}_{34} \boldsymbol{\Gamma}_{41} & 0
\end{array}\right]
$$

is obtained, as illustrated in Fig. 3(b). 
(a)

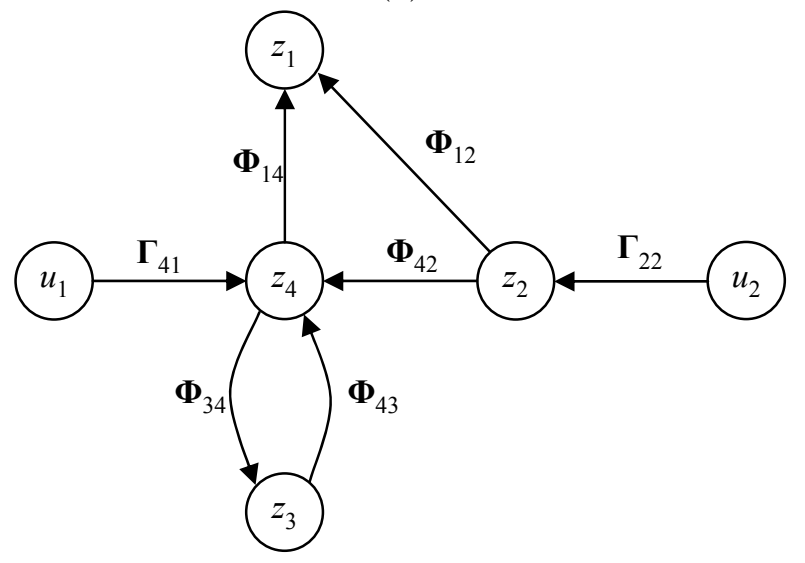

(b)

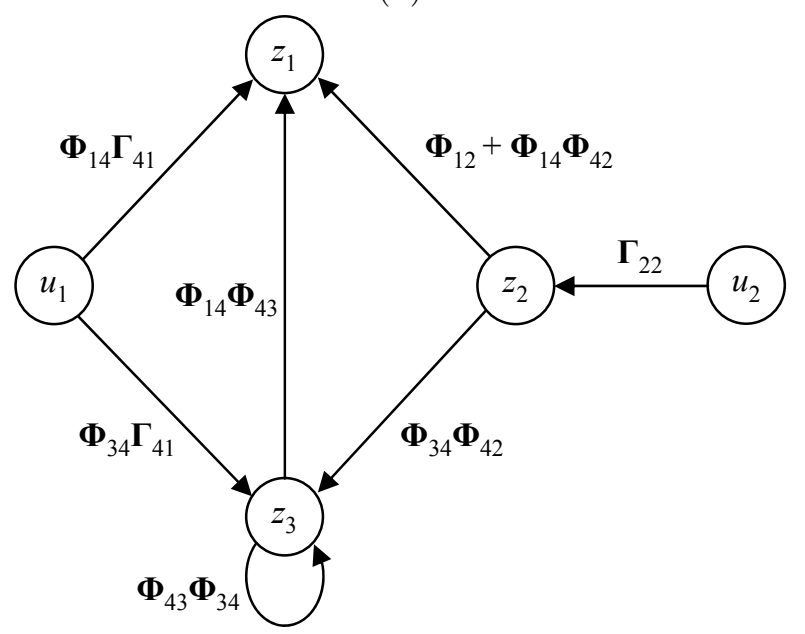

(c)

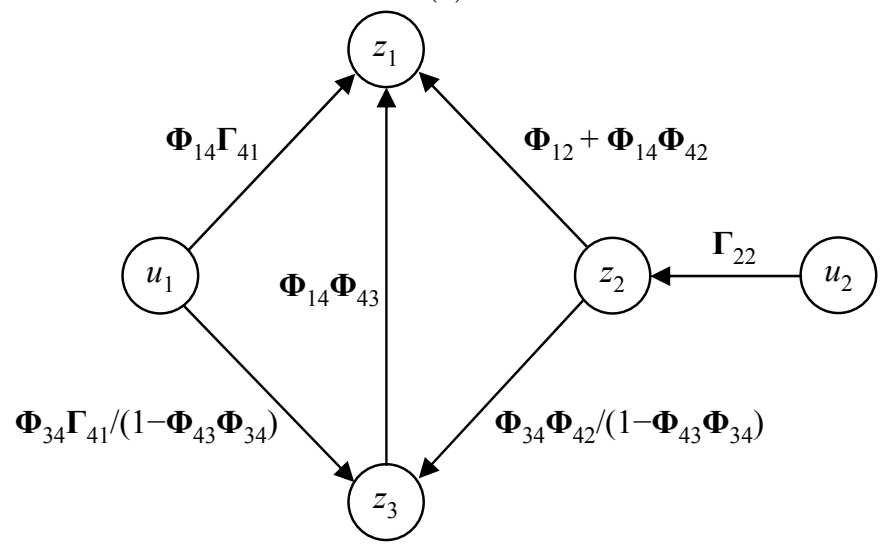

Fig. 3. Starting with the visualization in (a), node 4 is hidden resulting in the graph (b) and then the self reference of node 3 is removed, giving the visualization in $(\mathrm{c})$.

\subsection{Hiding arbitrary nodes}

Consider the partitioning of the interconnected system as $(\boldsymbol{\Phi}, \boldsymbol{\Gamma}, \boldsymbol{\Psi}, \boldsymbol{\Omega})$

$$
\begin{aligned}
{\left[\begin{array}{l}
z^{1} \\
z^{2}
\end{array}\right] } & =\left[\begin{array}{ll}
\boldsymbol{\Phi}^{11} & \boldsymbol{\Phi}^{12} \\
\boldsymbol{\Phi}^{21} & \boldsymbol{\Phi}^{22}
\end{array}\right]\left[\begin{array}{l}
z^{1} \\
z^{2}
\end{array}\right]+\left[\begin{array}{l}
\boldsymbol{\Gamma}^{1} \\
\boldsymbol{\Gamma}^{2}
\end{array}\right] u \\
y & =\left[\begin{array}{ll}
\boldsymbol{\Psi}^{1} & \boldsymbol{\Psi}^{2}
\end{array}\right]\left[\begin{array}{l}
z^{1} \\
z^{2}
\end{array}\right]+\boldsymbol{\Omega} u
\end{aligned}
$$

where $z^{2}=\left[\begin{array}{ll}0 & I_{m}\end{array}\right] z$ represents the last $m$ nodes. Assuming that $z^{\prime}=\Phi^{22} z^{\prime}+u^{\prime}$ is well-posed, we may now generalize (9) by isolating $z^{2}=\left(I-\boldsymbol{\Phi}^{22}\right)^{-1}\left(\boldsymbol{\Phi}^{21} z^{1}+\boldsymbol{\Gamma}^{2} u\right)$ and substituting this into the expressions for $z^{1}$ and $y$ to obtain

where

$$
\begin{aligned}
z^{1} & =\hat{\mathbf{\Phi}} z^{1}+\hat{\mathbf{\Gamma}} u \\
y & =\hat{\mathbf{\Psi}} z^{1}+\hat{\mathbf{\Omega}} u
\end{aligned}
$$

$$
\begin{aligned}
& \hat{\mathbf{\Phi}}=\boldsymbol{\Phi}^{11}+\boldsymbol{\Phi}^{12}\left(I-\boldsymbol{\Phi}^{22}\right)^{-1} \boldsymbol{\Phi}^{21} \\
& \hat{\boldsymbol{\Gamma}}=\boldsymbol{\Gamma}^{1}+\boldsymbol{\Phi}^{12}\left(I-\boldsymbol{\Phi}^{22}\right)^{-1} \boldsymbol{\Gamma}^{2} \\
& \hat{\mathbf{\Psi}}=\boldsymbol{\Psi}^{1}+\boldsymbol{\Psi}^{2}\left(I-\boldsymbol{\Phi}^{22}\right)^{-1} \boldsymbol{\Phi}^{21} \\
& \hat{\boldsymbol{\Omega}}=\boldsymbol{\Omega}+\boldsymbol{\Psi}^{2}\left(I-\boldsymbol{\Phi}^{22}\right)^{-1} \boldsymbol{\Gamma}^{2}
\end{aligned}
$$

This may be expressed as

$$
\begin{aligned}
{\left[\begin{array}{ll}
\hat{\boldsymbol{\Omega}} & \hat{\mathbf{\Psi}} \\
\hat{\boldsymbol{\Gamma}} & \hat{\mathbf{\Phi}}
\end{array}\right] } & =\left[\begin{array}{cc}
\boldsymbol{\Omega} & \boldsymbol{\Psi}^{1} \\
\boldsymbol{\Gamma}^{1} & \boldsymbol{\Phi}^{11}
\end{array}\right]+\left[\begin{array}{c}
\boldsymbol{\Psi}^{2} \\
\boldsymbol{\Phi}^{12}
\end{array}\right]\left(I-\boldsymbol{\Phi}^{22}\right)^{-1}\left[\begin{array}{ll}
\boldsymbol{\Gamma}^{2} & \boldsymbol{\Phi}^{21}
\end{array}\right] \\
& =\mathcal{F}\left(\left[\begin{array}{ll}
\boldsymbol{\Omega} & \boldsymbol{\Psi} \\
\boldsymbol{\Gamma} & \boldsymbol{\Phi}
\end{array}\right], I_{m}\right)
\end{aligned}
$$

To hide an arbitrary subset $\mathrm{N}$ of nodes, let us define the permutation matrix $T^{T}=\left[\begin{array}{ll}E^{T} & E^{\prime T}\end{array}\right]$ such that $z^{1}=E z$ is a vector containing only the nodes to be retained and $z^{2}=E^{\prime} z$ contains the nodes to be hidden. Requiring $E$ and $E^{\prime}$ to be on echelon form makes the definition unambiguous. Performing a linear transformation using $T$ as transformation matrix will now reorder the nodes so that the nodes to be hidden end up last in the new node vector $\hat{z}$. Thus, we may now define the operation $\mathcal{H}_{\mathrm{N}}$ of hiding an arbitrary set of nodes $\mathrm{N}$ as

$$
\mathcal{H}_{\mathrm{N}} \mathbf{Q}=\mathcal{F}\left(\left[\begin{array}{cc}
I_{q} & 0 \\
0 & T
\end{array}\right] \mathbf{Q}\left[\begin{array}{cc}
I_{p} & 0 \\
0 & T^{T}
\end{array}\right], I_{m}\right)
$$

From the construction of this hiding operation, it is clear that the input/output operators from $u$ to $y$ and and the remaining nodes $z^{1}$ are not altered and consequently Definition 3 is satisfied. The following theorem shows that it also satisfies Definition 4.

Theorem 1. The hiding operation (10) preserves the physical strucure in the sense of Definition 4.

Proof. Denote

$$
\overline{\mathbf{\Phi}}=T \boldsymbol{\Phi} T^{T}=\left[\begin{array}{ll}
\boldsymbol{\Phi}^{11} & \boldsymbol{\Phi}^{12} \\
\boldsymbol{\Phi}^{21} & \boldsymbol{\Phi}^{22}
\end{array}\right]
$$

Then $\hat{\boldsymbol{\Phi}}=\mathcal{F}\left(\overline{\boldsymbol{\Phi}}, I_{m}\right)=\boldsymbol{\Phi}^{11}+\boldsymbol{\Phi}^{12}\left(I-\boldsymbol{\Phi}^{22}\right)^{-1} \boldsymbol{\Phi}^{21}$ and $(I-\hat{\boldsymbol{\Phi}})^{-1}=\left(I-\boldsymbol{\Phi}^{11}-\boldsymbol{\Phi}^{12}\left(I-\boldsymbol{\Phi}^{22}\right)^{-1} \boldsymbol{\Phi}^{21}\right)^{-1}$. Also

$$
\begin{aligned}
& {\left[\begin{array}{cc}
E(I-\boldsymbol{\Phi})^{-1} E^{T} & * \\
* & *
\end{array}\right]=\left[\begin{array}{c}
E \\
E^{\prime}
\end{array}\right](I-\boldsymbol{\Phi})^{-1}\left[\begin{array}{ll}
E^{T} & E^{\prime T}
\end{array}\right]} \\
& =T(I-\boldsymbol{\Phi})^{-1} T^{T}=\left(I-T \boldsymbol{\Phi} T^{T}\right)^{-1} \\
& =\left[\begin{array}{cc}
I-\boldsymbol{\Phi}^{11} & -\boldsymbol{\Phi}^{12} \\
-\boldsymbol{\Phi}^{21} & I-\boldsymbol{\Phi}^{22}
\end{array}\right]^{-1} \\
& =\left[\begin{array}{cc}
\left(I-\boldsymbol{\Phi}^{11}-\boldsymbol{\Phi}^{12}\left(I-\boldsymbol{\Phi}^{22}\right)^{-1} \boldsymbol{\Phi}^{21}\right)^{-1} & * \\
* & *
\end{array}\right] \\
& =\left[\begin{array}{cc}
(I-\hat{\mathbf{\Phi}})^{-1} & * \\
* & *
\end{array}\right]
\end{aligned}
$$

where the fifth equality was obtained using the Schur complement for inverting partitioned matrices (Skogestad and Postlethwaite (2005)).

A consequence of the next theorem is that the hiding operation in (10) also satisfies Definition 5. 
Theorem 2. Let $\mathbf{Q}$ be an arbitrary visualization and let $\mathrm{N}$ and $\mathrm{M}$ be disjoint subsets of the nodes. Assume that the subsystems associated with $\mathrm{N}$ and $\mathrm{N} \cup \mathrm{M}$ are wellposed. Then the hiding operation (10) satisfies $\mathcal{H}_{\mathrm{M}} \mathcal{H}_{\mathrm{N}} \mathbf{Q}=$ $\mathcal{H}_{\mathrm{M} \cup \mathrm{N}} \mathbf{Q}$.

Proof. Let $N$ and $M$ be the number of elements in $\mathrm{N}$ and $\mathrm{M}$, respectively and let $T_{1}^{T}=\left[\begin{array}{ll}E_{1}^{T} E_{1}^{\prime T}\end{array}\right]$ be the permutation matrix for hiding nodes $\mathrm{N}$ and $T_{2}^{T}=\left[\begin{array}{ll}E_{2}^{T} & E_{2}^{\prime T}\end{array}\right]$ for hiding nodes $M$. The nodes retained after these two operations are then $E_{2} E_{1} z$ and the ones hidden are

$$
\left[\begin{array}{c}
E_{2}^{\prime} E_{1} \\
E_{1}^{\prime}
\end{array}\right] z
$$

Hiding $\mathrm{N}_{1} \cup \mathrm{N}_{2}$ in one operation thus requires the permutation matrix $T_{3}^{T}=\left[E_{3}^{T} E_{3}^{\prime T}\right]$ where $E_{3}=E_{2} E_{1}$, since this is already on echelon form and

$$
P E_{3}^{\prime}=\left[\begin{array}{c}
E_{2}^{\prime} E_{1} \\
E_{1}^{\prime}
\end{array}\right]
$$

for some permutation matrix $P$. After hiding nodes $\mathrm{N}$, the visualization $\mathcal{H}_{\mathrm{N}} \mathbf{Q}=\mathcal{F}\left(\hat{\mathbf{Q}}, I_{N}\right)$ is obtained, where

$$
\hat{\mathbf{Q}}=\left[\begin{array}{cc}
I_{q} & 0 \\
0 & T_{1}
\end{array}\right] \mathbf{Q}\left[\begin{array}{cc}
I_{p} & 0 \\
0 & T_{1}^{T}
\end{array}\right]
$$

Since the subsystem associated with $\mathrm{N} \cup \mathrm{M}$ is well-posed, we can hide these nodes simultaneously, resulting in

$$
\begin{aligned}
& \mathcal{H}_{\mathrm{M} \cup \mathrm{N}} \mathbf{Q}=\mathcal{F}\left(\left[\begin{array}{cc}
I_{q} & 0 \\
0 & T_{3}
\end{array}\right] \mathbf{Q}\left[\begin{array}{cc}
I_{p} & 0 \\
0 & T_{3}^{T}
\end{array}\right], I_{N+M}\right) \\
& =\mathcal{F}\left(\left[\begin{array}{cc}
I_{q} & 0 \\
0 & T_{3}
\end{array}\right] \mathbf{Q}\left[\begin{array}{cc}
I_{p} & 0 \\
0 & T_{3}^{T}
\end{array}\right], P^{T} P\right) \\
& =\mathcal{F}\left(\left[\begin{array}{cc}
I_{q+n-N-M} & 0 \\
0 & P
\end{array}\right]\left[\begin{array}{cc}
I_{q} & 0 \\
0 & T_{3}
\end{array}\right] \mathbf{Q}\left[\begin{array}{cc}
I_{p} & 0 \\
0 & T_{3}^{T}
\end{array}\right] \times\right. \\
& \left.\left[\begin{array}{cc}
I_{p+n-N-M} & 0 \\
0 & P^{T}
\end{array}\right], I_{N+M}\right)
\end{aligned}
$$

where Lemma 1(i) was used in the last equality. Now, since

$$
\begin{aligned}
& {\left[\begin{array}{cc}
I_{q+n-N-M} & 0 \\
0 & P^{T}
\end{array}\right]\left[\begin{array}{cc}
I_{q} & 0 \\
0 & T_{3}
\end{array}\right]=\left[\begin{array}{cc}
I_{q} & 0 \\
0 & E_{3} \\
0 & P E_{3}^{\prime}
\end{array}\right] } \\
= & {\left[\begin{array}{cc}
I_{q} & 0 \\
0 & E_{2} E_{1} \\
0 & E_{2}^{\prime} E_{1} \\
0 & E_{1}^{\prime}
\end{array}\right]=\left[\begin{array}{cc}
I_{q} & 0 \\
0 & T_{2} E_{1} \\
0 & E_{1}^{\prime}
\end{array}\right]=\left[\begin{array}{ccc}
I_{q} & 0 & 0 \\
0 & T_{2} & 0 \\
0 & 0 & I_{N}
\end{array}\right]\left[\begin{array}{cc}
I_{q} & 0 \\
0 & T_{1}
\end{array}\right] }
\end{aligned}
$$

we have

$$
\begin{aligned}
& \mathcal{H}_{\mathrm{M} \cup \mathrm{N}} \mathbf{Q} \\
& =\mathcal{F}\left(\left[\begin{array}{ccc}
I_{q} & 0 & 0 \\
0 & T_{2} & 0 \\
0 & 0 & I_{N}
\end{array}\right] \hat{\mathbf{Q}}\left[\begin{array}{ccc}
I_{p} & 0 & 0 \\
0 & T_{2}^{T} & 0 \\
0 & 0 & I_{N}
\end{array}\right], I_{N+M}\right) \\
& =\mathcal{F}\left(\mathcal{F}\left(\left[\begin{array}{ccc}
I_{q} & 0 & 0 \\
0 & T_{2} & 0 \\
0 & 0 & I_{N}
\end{array}\right] \hat{\mathbf{Q}}\left[\begin{array}{ccc}
I_{p} & 0 & 0 \\
0 & T_{2}^{T} & 0 \\
0 & 0 & I_{N}
\end{array}\right], I_{N}\right), I_{M}\right) \\
& =\mathcal{F}\left(\left[\begin{array}{cc}
I_{q} & 0 \\
0 & T_{2}
\end{array}\right] \mathcal{F}\left(\hat{\mathbf{Q}}, I_{N}\right)\left[\begin{array}{cc}
I_{p} & 0 \\
0 & T_{2}^{T}
\end{array}\right], I_{M}\right) \\
& =\mathcal{H}_{\mathrm{M}} \mathcal{H}_{\mathrm{N}} \mathbf{Q}
\end{aligned}
$$

where Lemma 1(ii) was used in the second equality and Lemma 1(i) in the third.

\subsection{Eliminating self references}

A node that depends on itself (i.e. a nonzero diagonal element in $\boldsymbol{\Phi}$ ) may be disadvantageous for the visual interpretation of the interconnected system. Given such an element $\boldsymbol{\Phi}_{i i}$, it is straightforward to remove it by moving the term $\boldsymbol{\Phi}_{i i} z_{i}$ to the left hand side of (2) and isolating $z_{i}$ as

$$
z_{i}=\frac{1}{1-\boldsymbol{\Phi}_{i i}}\left(\boldsymbol{\Phi}_{i} z-\boldsymbol{\Phi}_{i i} z_{i}+\boldsymbol{\Gamma}_{i} u\right)
$$

where $\boldsymbol{\Phi}_{i}$ and $\boldsymbol{\Gamma}_{i}$ are the $i$ th row of $\boldsymbol{\Phi}$ and $\boldsymbol{\Gamma}$, respectively. The requirement for this operation to be defined is that the subsystem associated with node $i$ is well-posed.

Example 3. In Example 2, a self reference appeared on the third node when the fourth node was hidden. Eliminating this self reference using (11) results in

$$
\begin{aligned}
\boldsymbol{\Phi}^{\prime \prime} & =\left[\begin{array}{ccc}
0 & \boldsymbol{\Phi}_{12}+\boldsymbol{\Phi}_{14} \boldsymbol{\Phi}_{42} & \boldsymbol{\Phi}_{14} \boldsymbol{\Phi}_{43} \\
0 & 0 & 0 \\
0 & \frac{\boldsymbol{\Phi}_{34} \boldsymbol{\Phi}_{42}}{1-\boldsymbol{\Phi}_{43} \boldsymbol{\Phi}_{34}} & 0
\end{array}\right] \\
\boldsymbol{\Gamma}^{\prime \prime} & =\left[\begin{array}{cc}
\boldsymbol{\Phi}_{14} \boldsymbol{\Gamma}_{41} & 0 \\
0 & \boldsymbol{\Gamma}_{22} \\
\frac{\boldsymbol{\Phi}_{34} \boldsymbol{\Gamma}_{41}}{1-\boldsymbol{\Phi}_{43} \boldsymbol{\Phi}_{34}} & 0
\end{array}\right]
\end{aligned}
$$

as in Fig. 3(c).

It is straightforward to generalize this into elimination of self references for a set $\mathrm{N}$ of nodes by defining $D$ as a matrix where every element is 1 except zeros at the diagonal elements corresponding to the self references to be eliminated. Thus, provided that the scalar subsystem associated with each node in $\mathrm{N}$ is well-posed, we may define the operator $\mathcal{E}_{\mathrm{N}}$ eliminating the self references for nodes $\mathrm{N}$ as

$$
\mathcal{E}_{\mathrm{N}}\left[\begin{array}{cc}
\boldsymbol{\Omega} & \boldsymbol{\Psi} \\
\boldsymbol{\Gamma} & \boldsymbol{\Phi}
\end{array}\right]=\left[\begin{array}{cc}
I_{q} & 0 \\
0 & (I-(1-D) \circ \boldsymbol{\Phi})^{-1}
\end{array}\right]\left[\begin{array}{cc}
\boldsymbol{\Omega} & \boldsymbol{\Psi} \\
\boldsymbol{\Gamma} & D \circ \boldsymbol{\Phi}
\end{array}\right]
$$

where $D \circ \boldsymbol{\Phi}$ removes the diagonal element $\boldsymbol{\Phi}_{i i}$ in $\boldsymbol{\Phi}$ corresponding to each node in $\mathrm{N}$ while the factor $(I-$ $(1-D) \circ \boldsymbol{\Phi})^{-1}$ provides the multiplication by $\frac{1}{1-\boldsymbol{\Phi}_{i i}}$ of the corresponding rows in $\boldsymbol{\Phi}$ and $\boldsymbol{\Gamma}$.

Example 4. A linearized state-space model of the tank process in Fig. 2(a) with the tank levels as state variables is

$$
\begin{aligned}
& \dot{z}_{1}=-\frac{1}{T} z_{1}+\frac{k}{T} u \\
& \dot{z}_{2}=-\frac{1}{T} z_{2}+\frac{1}{T} z_{1}
\end{aligned}
$$

which may be directly translated into a visualization with

$$
\Phi(s)=\left[\begin{array}{cc}
-\frac{1}{T s} & 0 \\
\frac{1}{T s} & -\frac{1}{T s}
\end{array}\right], \quad \Gamma(s)=\left[\begin{array}{c}
\frac{k}{T s} \\
0
\end{array}\right]
$$

as illustrated in Fig. 4(a) When the self references are eliminated using (12), the visualization in (7), illustrated in Fig. 4(b) is obtained.

Theorem 3. Let $\mathbf{Q}$ be an arbitrary visualization and let N, M be subsets of the nodes. Assume that the scalar subsystems associated with each node in $\mathrm{N} \cup \mathrm{M}$ are well-posed. Then the elimination operation (12) satisfies $\mathcal{E}_{\mathrm{M}} \mathcal{E}_{\mathrm{N}} \mathbf{Q}=\mathcal{E}_{\mathrm{N} \cup \mathrm{M}} \mathbf{Q}$. 
(a)

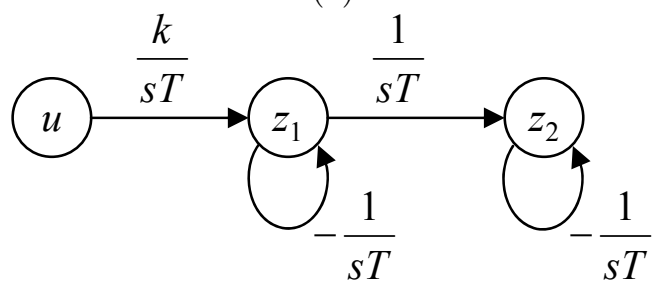

(b)

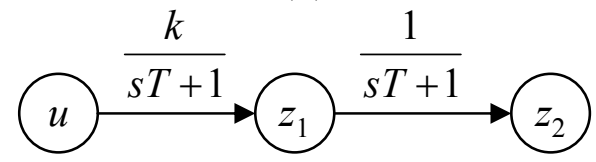

Fig. 4. (a) Visualization of the tank process on state-space form. (b) Visualization with self-references eliminated

Proof. Elimination of the self reference for a node $z_{i}$ only affects and is affected by row $i$ in $\boldsymbol{\Gamma}$ and $\boldsymbol{\Phi}$. Thus, eliminations can be performed simultaneously or in arbitrary order.

The following theorem will be the final piece required. In combination with Theorem 2 and Theorem 3, it will make it possible to show that for any number of hiding operations $\mathcal{H}_{\mathrm{M}_{1}}, \ldots, \mathcal{H}_{\mathrm{M}_{M}}$ and self reference eliminations $\mathcal{E}_{\mathrm{N}_{1}}, \ldots, \mathcal{E}_{\mathrm{N}_{N}}$ the same result is obtained regardless of the order of these operations as long as the last operation is elimination of the self references $\mathrm{N}=\bigcup_{i=1}^{N} \mathrm{~N}_{i} \backslash \bigcup_{i=1}^{M} \mathrm{M}_{i}$ and that all the relevant subsystems are well-posed.

Theorem 4. Let $\mathbf{Q}$ be an arbitrary visualization and let $\mathrm{N}, \mathrm{M}$ be subsets of the nodes. Assume that the subsystems associated with $\mathrm{M}$ and each node in $\mathrm{N}$ are well-posed. Then the operations defined in (10) and (12) satisfy $\mathcal{E}_{\mathrm{N} \backslash \mathrm{M}} \mathcal{H}_{\mathrm{M}} \mathcal{E}_{\mathrm{N}} \mathbf{Q}=\mathcal{E}_{\mathrm{N} \backslash \mathrm{M}} \mathcal{H}_{\mathrm{M}} \mathbf{Q}$.

Proof. Omitted for brevity.

\section{THE LOOP GAIN INDEX (LGI)}

Here, we propose a measure for the importance of each edge with respect to the dynamical behavior of the interconnected system. The aspect that is considered is whether the edge is part of a significant feedback loop. It is well known that closing a loop may alter the behavior of a dynamic system, particularly if the loop gain (with respect to some signal norm) is large. Indeed, if the gain is larger than unity then instability may occur. We will therefore introduce the loop gain array (LGA) as the loop gains of the elements in $\boldsymbol{\Phi}$. For an element $\boldsymbol{\Phi}_{j i}$ this is constructed by introducing the auxiliary input $\zeta_{i}$ with $\boldsymbol{\Phi}_{j i}$ from $\zeta_{i}$ to $z_{j}$ and the auxiliary output $\xi_{j}$ to $z_{i}$. Then element $j, i$ is removed and element $j, i$ of the LGA is obtained by taking the input/output system from $\zeta_{i}$ to $\xi_{j}$, see Fig. 5(a).

Proposition 1. Element $j, i$ of the loop gain array is

$$
\boldsymbol{\Lambda}_{j i}=\frac{\boldsymbol{\Phi}_{j i}\left[(I-\boldsymbol{\Phi})^{-1}\right]_{i j}}{1+\boldsymbol{\Phi}_{j i}\left[(I-\mathbf{\Phi})^{-1}\right]_{i j}}
$$

Proof. The effect of removing $\boldsymbol{\Phi}_{j i}$ can also be obtained by cancelling its effect by introducing the gain $-\boldsymbol{\Phi}_{j i}$ from $z_{i}$ to $z_{j}$. In Fig. 5(b), this is accomplished with the auxiliary signal $x_{i}$, to which the input $\zeta_{i}$ is also connected. The input/output relation from $\zeta_{i}\left(\right.$ or $x_{i}$ ) to $\xi_{j}$ with the edge (a)

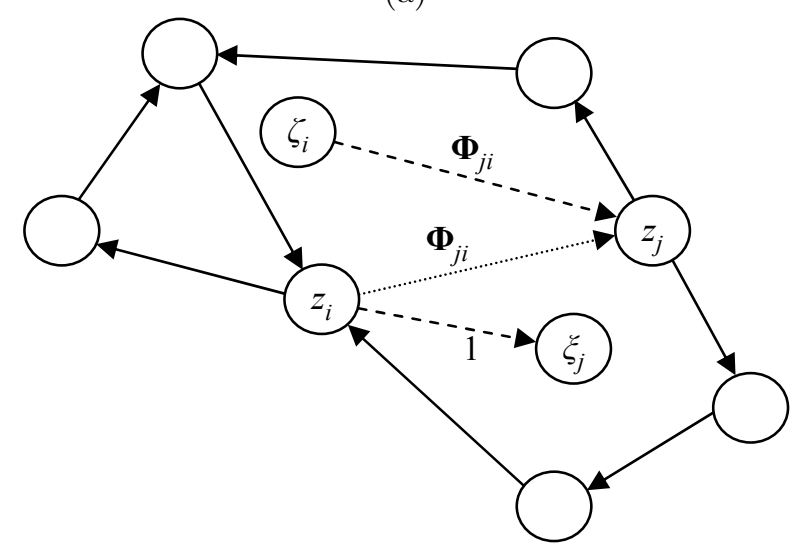

(b)

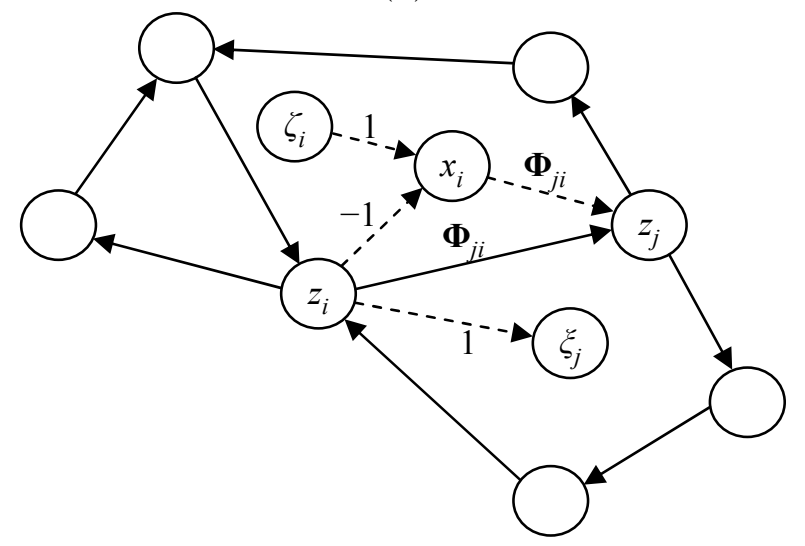

(c)

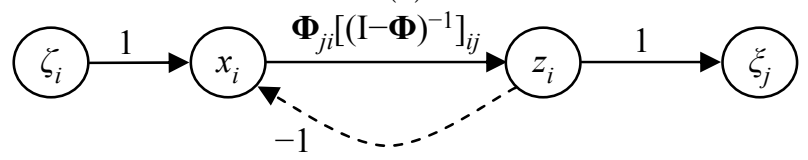

Fig. 5. The loop gain for element $\boldsymbol{\Phi}_{j i}$ is found by removing the dotted line and introducing the dashed lines as in (a). An equivalent operation is to introduce the dashed lines in (b) which, in turn, may be redrawn as (c).

-1 absent is obtained by introducing the input $\zeta_{i}$ through $\boldsymbol{\Gamma}=e_{j} \boldsymbol{\Phi}_{j i}$ and extracting the output $\xi_{j}$ with $\boldsymbol{\Psi}=e_{i}^{T}$ which results in $\boldsymbol{\Psi}(I-\boldsymbol{\Phi})^{-1} \boldsymbol{\Gamma}=\boldsymbol{\Phi}_{j i}\left[(I-\boldsymbol{\Phi})^{-1}\right]_{i j}$. The graph can thus be simplified to the appearance of Fig. 5(c) where the edge -1 from Fig. 5(b) is now introduced. Recognizing this as a unit feedback gives (13).

To evaluate the LGA, we propose to use the $\mathrm{H}_{\infty}$-norm of its elements, thus introducing the Loop Gain Index LGI as

$$
\left[J_{L}\right]_{j i}=\left\|\boldsymbol{\Lambda}_{j i}\right\|_{\infty}
$$

It should be noted that the LGI only considers whether a particular edge contributes to a significant loop or not. An edge may thus be important even if its LGI is low, e.g. if it is the only way for a specific input to affect the outputs.

\subsection{Numerical example}

A numerical example to illustrate the LGI is given here. For comparison, the $\mathrm{H}_{\infty}$-norm of each edge in $\boldsymbol{\Phi}$, termed 
the Norm Index $J_{N}$, is also calculated, i.e. $\left[J_{N}\right]_{i j}=$ $\left\|\boldsymbol{\Phi}_{i j}\right\|_{\infty}$.

Example 5. Let

$$
\begin{aligned}
\Gamma(s) & =\left[\begin{array}{llll}
1 & 0 & 0 & 0
\end{array}\right]^{T} \\
\Phi(s) & =\left[\begin{array}{cccc}
0 & 0 & 0 & \frac{-0.1}{s+1} \\
\frac{3}{2 s+1} & 0 & 0 & \\
\frac{1}{s+1} & \frac{3}{3 s+1} & 0 & 0 \\
0 & 0 & \frac{1}{4 s+1} & 0
\end{array}\right] \\
\Psi(s) & =I
\end{aligned}
$$

The LGI is

$$
J_{L}=\left[\begin{array}{cccc}
0 & 0 & 0 & 0.2441 \\
0.2071 & 0 & 0 & 0 \\
0.0275 & 0.2071 & 0 & 0 \\
0 & 0 & 0.2441 & 0
\end{array}\right]
$$

to be compared to the norm index

$$
J_{N}=\left[\begin{array}{cccc}
0 & 0 & 0 & 0.0707 \\
1.5000 & 0 & 0 & 0 \\
0.7071 & 1.2247 & 0 & 0 \\
0 & 0 & 0.3536 & 0
\end{array}\right]
$$

Here, it can be noted that the element in position 3,1 which is quite large with respect to the $\mathrm{H}_{\infty}$-norm turns out to be insignificant in terms of the LGI and that element 1,4 which is very small in terms of the $\mathrm{H}_{\infty}$-norm gives a large LGI. The conclusion is that $\boldsymbol{\Phi}_{14}$ is important for the dynamic behavior while $\boldsymbol{\Phi}_{31}$ is not. This is confirmed by the step response shown in Fig. 6. Clearly, neglecting element 1,4 alters the step response much more than neglecting element 3,1 .

\section{CONCLUSIONS}

The concept of visualization of a system by representing it as a signal flow graph was introduced. An operation for hiding nodes of a visualization while preserving its dynamics and physical structure in a certain sense was was defined. Also, an example of an analysis tool was introduced, termed the Loop Gain Index, intended for determining the importance of each edge of the signal flow graph in terms of the dynamics of the input/output system.

\section{ACKNOWLEDGMENTS}

The author wishes to thank the Hjalmar Lundbohm research center $(H L R C)$ funded by LKAB and the Swedish Governmental Agency for Innovation Systems (VINNOVA) for financing this research.

\section{REFERENCES}

Castaño, M. and Birk, W. (2009). New methods for structural and functional analysis of complex processes. In IEEE International Conference on Control Applications: CCA '09, St. Petersburg, Russia, 487-494.

Ding, L., Gustafsson, T., and Johansson, A. (2007). Model parameter estimation of simplified linear models for a continuous paper pulp digester. Journal of Process Control, 17(2), 115-127.
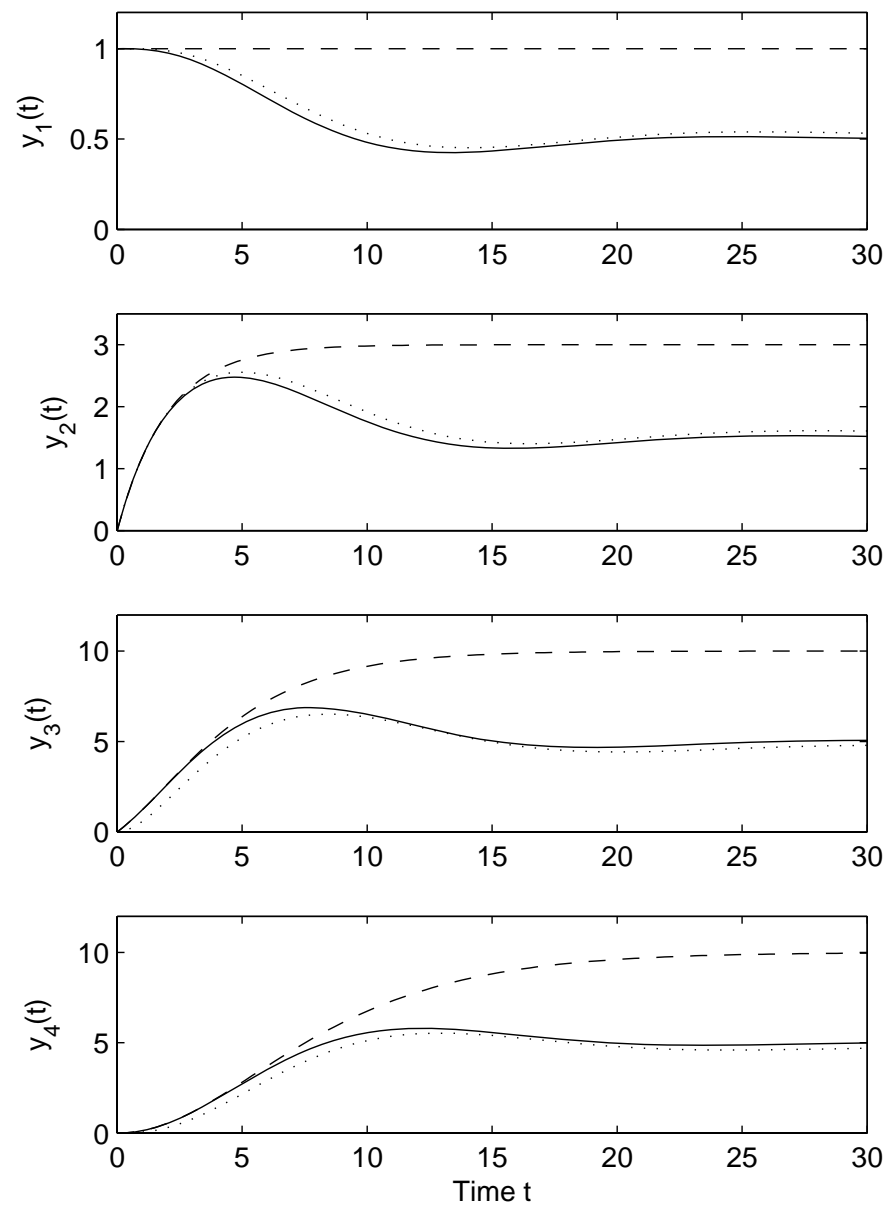

Fig. 6. Step response for the system in Example 5. Solid line shows the original system while the dashed and dotted lines represents the cases when $\boldsymbol{\Phi}_{14}$ and $\boldsymbol{\Phi}_{31}$ is neglected, respectively.

Mason, S.J. (1953). Feedback theory - Some properties of signal flow graphs. Proceedings of the IRE, 1144-1156.

Sezer, M.E. and Šiljak, D.D. (1986). Nested $\varepsilon$ decompositions and clustering of complex systems. Automatica, 22(3), 321-331.

Skogestad, S. and Postlethwaite, I. (2005). Multivariable Feedback Control. Wiley.

Vidyasagar, M. (1980). On the well-posedness of largescale interconnected systems. IEEE Transactions on Automatic Control, 25(3), 413-421.

Zečević, A.I. and Šiljak, D.D. (2005). A decompositionbased control strategy for large, sparse dynamic systems. Mathematical problems in engineering, (1), 33-48. 